\title{
PERCEPÇÃO E CONHECIMENTO DE PROFISSIONAIS DE UNIDADES BÁSICAS DA SAÚDE SOBRE ACESSIBILIDADE DAS PESSOAS COM DEFICIÊNCIA
}

\author{
PERCEPCIÓN Y CONOCIMIENTO DE PROFESIONALES DE LA UNIDADES \\ BÁSICA DE SALUD SOBRE ACCESIBILIDAD DE PERSONAS CON DISCAPACIDAD
}

\author{
PERCEPTION AND KNOWLEDGE OF BASIC HEALTH UNITS PROFESSIONALS \\ ON ACCESSIBILITY FOR PEOPLE WITH DISABILITIES
}

\author{
Bruna Martins Grassi SEDLMAIER ${ }^{1}$ \\ Denise Machado MOURÃO ${ }^{2}$ \\ Cristiane Gomes FERREIRA ${ }^{3}$ \\ Carla Ladeira Gomes da SILVEIRA ${ }^{4}$ \\ Grasiely Faccin BORGES ${ }^{5}$
}

RESUMO: O objetivo deste estudo é avaliar a percepção e o conhecimento dos profissionais de Unidades Básicas de Saúde (UBSs) sobre a acessibilidade das Pessoas com Deficiência (PcD) e sua assistência nos serviços. A pesquisa é um estudo transversal, quanti-qualitativo em que foi realizada entrevista semiestruturada com 30 questões. Com base nas entrevistas de 53 participantes, identificou-se que quase metade alegou não conhecer a legislação sobre os direitos das $\mathrm{PcD}, 44 \%$ relataram ter alguma dificuldade ou dúvida sobre o atendimento da $\mathrm{PcD}, 45,3 \%$ afirmaram que não havia distinção no acolhimento da $\mathrm{PcD}$ aos demais usuários e apenas $37,7 \%$ afirmaram que a PcD tinha prioridade no acolhimento. Concluiu-se, portanto, que o conhecimento dos profissionais das UBSs sobre acessibilidade e assistência da PcD nos serviços de saúde precisa ser aprimorado, para que o cuidado seja resolutivo, de forma a valorizar a autonomia e independência das PcD.

PALAVRAS-CHAVE: Pessoas com deficiência. Estratégia saúde da família. Acesso aos serviços de saúde. Assistência integral à saúde.

RESUMEN: Objetivo: evaluar la percepción y el conocimiento de los profesionales de la Unidades Básicas de Salud (UBSs) sobre la accesibilidad de las personas con discapacidad

${ }^{1}$ Universidade Federal do Sul da Bahia (UFSB), Teixeira de Freitas - BA - Brasil. Graduanda no curso de Medicina no Centro de Formação em Ciências da Saúde. ORCID: https://orcid.org/0000-0002-4069-1075. Email: brunasedlmaier@live.com

${ }^{2}$ Universidade Federal do Sul da Bahia (UFSB), Teixeira de Freitas - BA - Brasil. Professora Adjunta no Centro de Formação em Ciências da Saúde. Doutorado em Ciência e Tecnologia de Alimentos (UFV). ORCID: https://orcid.org/0000-0002-7265-6899. E-mail: dmmourao@gmail.com

${ }^{3}$ Universidade do Estado da Bahia (UNEB), Teixeira de Freitas - BA - Brasil. Professora Assistente no Departamento de Educação. Mestrado em Educação e Contemporaneidade (UNEB). ORCID: https://orcid.org/0000-0003-4697-6423.E-mail: crisgfe.inclusao@gmail.com

${ }^{4}$ Universidade Federal do Sul da Bahia (UFSB), Teixeira de Freitas - BA - Brasil. Graduanda no curso de Medicina no Centro de Formação em Ciências da Saúde. ORCID: https://orcid.org/0000-0002-1935-7486. Email: carlalgsilveira@gmail.com

${ }^{5}$ Universidade Federal do Sul da Bahia (UFSB), Itabuna - BA - Brasil. Professora Adjunta da Pós-Graduação em Saúde, Ambiente e Biodiversidade. Doutorado em Ciências do Desporto (UC) - Portugal. ORCID: https://orcid.org/0000-0002-5771-6259. E-mail: grasiely.borges@gmail.com

Temas em Educ. e Saúde, Araraquara, v. 17, n. 00, e021003, 2021 e-ISSN 2526-3471 
(PcD) y su asistencia en los servicios. Métodos: este es un estudio transversal, cuantitativo y cualitativo. Se realizó una entrevista semiestructurada con 30 preguntas. Resultados: participaron 53 encuestados, casi la mitad de los participantes afirmó no conocer la legislación sobre los derechos de las PcD, el 44\% informó tener alguna dificultad o duda sobre el cuidado de las PcD, el 45,3\% declaró que no habia distinción en dar la bienvenida a los demás usuarios y solo el 37,7\% declaró que el PcD tenía prioridad en el alojamiento. Conclusión: es necesario mejorar el conocimiento de los profesionales de UBSs sobre accesibilidad y asistencia para PcD en los servicios de salud, para que la atención sea resolutiva, a fin de valorar la autonomía e independencia de las PcD.

PALABRAS CLAVE: Personas con discapacidad. Estrategia de salud familiar. Accesibilidad a los servicios de salud. Atención integral de salud.

ABSTRACT: The aim of this study is to evaluate the perception and knowledge of Basic Health Units (UBSS) professionals about accessibility of People with Disabilities $(P w D)$ and their assistance in services. The research is a cross-sectional, quantitative and qualitative study. A semi-structured interview was conducted with 30 questions. Based on the interviews of 53 participants, it was identified that almost half of the participants did not know the legislation on the rights of the PwD, 44\% reported having some difficulty or doubt about the care of the DP, 45.3\% stated that there was no distinction in welcoming the PwD to other users and only 37.7\% stated that the PwD had priority in hosting. It was concluded, therefore, that the knowledge of UBSs professionals about accessibility and assistance of $P w D$ in health services needs to be improved, so that care is resolute, in order to value the autonomy and independence of $P w D$.

KEYWORDS: Disabled persons. Family health strategy. Health services accessibility. Comprehensive health care.

\section{Introdução}

A Atenção Básica consiste na porta de entrada preferencial do Sistema Único de Saúde para todos os usuários. A Estratégia Saúde da Família (ESF) favorece o acesso à assistência à saúde e é essencial para a consolidação da Atenção Básica (BRASIL, 2017). O acesso às ESF, como em todos os espaços arquitetônicos e urbanísticos, deve envolver condições para que as pessoas com deficiência, de modo a atender com equidade todos os cidadãos.

A acessibilidade pode ser conceituada na possibilidade e condição de alcance, percepção e entendimento para utilização segura e com autonomia de todos os espaços urbanos, incluindo mobiliários, transportes, comunicação e serviços ao público, por PcD ou com mobilidade reduzida (ASSOCIAÇÃO BRASILEIRA DE NORMAS TÉCNICAS, 2015). A acessibilidade do usuário representa o ajuste entre as características dos recursos da atenção 
à saúde e os da população, ela é a facilidade em utilizar os serviços de forma satisfatória (PEDRAZA et al., 2018).

No Plano Nacional de Direitos da PcD de 2011 foram estabelecidos os eixos de acesso à educação, à atenção à saúde, à inclusão social e à acessibilidade, incentivando políticas articuladas e intersetoriais para garantir a inclusão das PcD no SUS, com foco na integralidade do cuidado (BRASIL, 2011). Também, a portaria $\mathrm{n}^{\circ} 793$ de 2012 instituiu a rede de cuidados à $\mathrm{PcD}$, com a criação, ampliação e articulação de pontos estratégicos na atenção à PcD no Sistema Único de Saúde (SUS). Destaca-se neste documento o estímulo a livre e espontânea procura destes usuários até as unidades, de maneira autônoma e independente (BRASIL, 2012). Posteriormente, a lei 13146 de 2015 - lei brasileira de inclusão da PcD veio com a previsão do atendimento prioritário, com ênfase às políticas públicas em saúde (BRASIL, 2015).

Apesar de existir uma legislação quanto ao cuidado da PcD no Brasil, estas leis e decretos precisam ser garantidas na vida cotidiana destas pessoas e, em especial, na área de saúde. Alguns estudos buscaram investigar esta temática (ALBUQUERQUE et al., 2014; BEZERRA; SILVA; MAIA, 2015; CASTRO et al., 2011; CRUZ et al., 2019; MAIA et al., 2009; MOCELIN et al., 2017; RIBEIRO et al., 2015; SILVA et al., 2015), tendo um predomínio na investigação da acessibilidade física em unidades de saúde ou na abordagem de $\mathrm{PcD}$ junto a outros grupos com acesso prejudicado, como pessoas não alfabetizadas e idosos.

Para além das barreiras arquitetônicas, que comprometem o direito de acesso do cidadão, pela falta de acessibilidade nos serviços de saúde e interferem diretamente a sua qualidade de vida (CRUZ et al., 2019; SILVA et al., 2015). As percepções e as competências dos profissionais na assistência à $\mathrm{PcD}$, visando a uma assistência integral a esse público é de grande importância nesse processo (BEZERRA; SILVA; MAIA, 2015; MAIA et al., 2009).

As condutas equivocadas e que prejudicam a acessibilidade devem ser discutidas entre todos os trabalhadores da unidade e gestão, a fim de descobrir as causas que têm levado aqueles que atuam, principalmente, na recepção, às atitudes que excluem o usuário da porta de entrada do SUS. O estudo do conhecimento e percepção dos trabalhadores que atuam nas UBSs pode possibilitar a criação e implementação de estratégias que modifiquem o processo de trabalho e qualifique o acolhimento do PcD. Diante disso, o presente trabalho objetivou avaliar a percepção e o conhecimento dos trabalhadores de Unidades Básicas de Saúde sobre acessibilidade da PcD e sua assistência nos serviços em uma cidade do extremo sul da Bahia. 


\section{Métodos}

Trata-se de um estudo transversal, de caráter quanti-qualitativo, realizado junto as Unidades Básicas de Saúde no município de Teixeira de Freitas, Bahia.

O universo de estudo foram trabalhadores que atuavam em Unidades Básicas de Saúde, projetadas e construídas para funcionar com esse fim, sendo excluídas todas as unidades com espaços alugados e adaptados. Esse critério foi aplicado visando minimizar discrepâncias nas respostas devido à influência arquitetônica e à falta de adequação das unidades, visto que as unidades projetadas para o funcionamento das ESF deveriam seguir a Norma Brasileira ABNT-NBR 9050/2015 (ASSOCIAÇÃO BRASILEIRA DE NORMAS TÉCNICAS, 2015).

A coleta de dados foi realizada em 13 ESFs, todas localizadas no meio urbano do município de Teixeira de Freitas e nas cindo regiões de cobertura da Atenção Básica. Foi utilizado um questionário para verificar informações sobre o conhecimento e as percepções dos profissionais, por meio de uma entrevista semiestruturada com 30 questões, sendo duas fechadas e as demais questões abertas. Em algumas questões foram solicitados exemplos e justificativas, para confirmação das respostas.

A entrevista foi realizada com pelo menos quatro trabalhadores de cada unidade, que estavam em contato constante com a comunidade, passavam mais tempo na unidade e participavam do acolhimento, sendo convidados a participar, prioritariamente: recepcionistas, agentes comunitários de saúde, técnicos de enfermagem ou enfermeiros das unidades visitadas. Os critérios de inclusão para participar da entrevista foram: exercer a função há pelo menos três meses e ter atendido pelo menos uma $\mathrm{PcD}$ na unidade básica de saúde até o momento da coleta de dados. Os critérios de exclusão foram: trabalhadores ausentes por quaisquer motivos no momento da pesquisa ou não concordar em assinar o TCLE.

As questões abordadas na entrevista foram agrupadas de acordo com a temática de cada uma das perguntas, caracterizando: o perfil dos entrevistados (sexo, faixa etária, escolaridade e cargo), conhecimento dos entrevistados sobre legislação relacionada à $\mathrm{PcD}$, atendimento, cuidado e comunicação, percepção sobre acessibilidade e inclusão nas ESF, percepção dos entrevistados sobre a PcD.

No momento da entrevista, as respostas dos participantes foram manualmente registradas e questionário de papel e, posteriormente, tabuladas em planilha Excel. As variáveis qualitativas foram interpretadas pela análise de discurso das entrevistas, sendo realizada uma categorização de acordo com as palavras-chaves utilizadas pelos profissionais. 
Os dados foram representados por percentuais e apresentados na forma de tabelas. O estudo seguiu as Normas e Diretrizes Regulamentadoras da Pesquisa envolvendo Seres Humanos Resolução CNS 466/2012 e 510/2016, sendo aprovado pelo Comitê de Ética em Pesquisa da Universidade Estadual de Santa Cruz em 22 de setembro de 2017, $\mathrm{n}^{\circ}$ CAAE 72192317.0 .0000 .5526 .

\section{Resultados}

Responderam ao questionário 53 participantes, dentre eles 27 agentes comunitários de saúde, 10 enfermeiros, 11 recepcionistas e cinco (5) técnicos de enfermagem das unidades básicas de saúde estudadas. Destes a maior parte (81,2\%) era do sexo feminino, tinha entre 35 a 39 anos (28,3\%) e possuía nível médio completo (41,5\%) (Tabela 1).

Tabela 1 - Perfil dos entrevistados do estudo $(\mathrm{n}=53)$. Teixeira de Freitas, BA, Brasil, 2017.

\begin{tabular}{lrr}
\hline Variáveis & n & \% \\
\hline Sexo & & \\
\hline Feminino & 43 & 81,2 \\
Masculino & 10 & 18,8 \\
\hline Faixa etária & & \\
\hline 20 a 24 & 1 & 1,9 \\
25 a 29 & 7 & 13,2 \\
30 a 34 & 8 & 15,1 \\
35 a 39 & 15 & 28,3 \\
40 a 44 & 7 & 13,2 \\
45 a 49 & 10 & 18,8 \\
50 a 54 & 3 & 5,7 \\
55 a 59 & 1 & 1,9 \\
60 a 64 & 0 & 0,0 \\
65 a 69 & 1 & 1,9 \\
\hline Escolaridade & & \\
\hline Ensino Fundamental Completo & 2 & 3,8 \\
Nível Médio Completo & 22 & 41,5 \\
Nível Técnico Completo & 11 & 20,7 \\
Ensino Superior Completo & 12 & 22,7 \\
Especialização Completa & 6 & 11,3 \\
\hline
\end{tabular}

Fonte: Elaborado pelas autoras

O tempo médio de atuação na profissão dos entrevistados foi de 10 anos, obtendo-se valores mínimo e máximo de 7 meses e de 20 anos, respectivamente. Os trabalhadores 
participantes tiveram o tempo médio de atuação nas unidades, em que estavam exercendo função, de cinco anos e dois meses, sendo os valores mínimo e máximo de três meses e de 18 anos, respectivamente.

Os participantes relataram conhecer a legislação (53\%) (Tabela 2). Daqueles que afirmaram conhecer a legislação, apenas dois entrevistados não conseguiram citar exemplos condizentes com as leis. Dos que alegaram conhecer, as mais citadas foram: prioridade prevista para essa população; direito à saúde; aposentadoria; acessibilidade nos transportes e ambientes físicos; além das cotas reservadas para trabalho em empresas.

Os participantes informaram que a PcD teria prioridade no atendimento na ESF (77,4\%) (Tabela 2). Entretanto, sobre a questão de prioridade no atendimento das PcD nas ESF, pode-se observar três tipos de categorias de respostas distintas: (1) atendimento prioritário 'tradicional', (2) atendimento por ordem de chegada, e (3) prioridade pelo nível de risco.

Os participantes da pesquisa afirmaram que o acolhimento de PcD nas ESF não tinha distinção em relação aos demais usuários (45,3\%), que não havia diferença na relação/atendimento da $\mathrm{PcD}$, pelo tipo de deficiência (49,1\%), que utilizavam estratégia(s), como gestos ou mímicas, escrita, leitura labial e fala pausada para comunicação com a PcD no momento do atendimento, e que surdos ou PcD intelectual têm a necessidade de comparecer na ESF com um acompanhante (68\%) (Tabela 2).

Tabela 2 - Frequência de respostas do conhecimento e percepções dos trabalhadores das Unidades Básicas de Saúde sobre o atendimento da Pessoa com Deficiência ( $\mathrm{n}=53)$.

Teixeira de Freitas, BA, Brasil, 2017.

\begin{tabular}{lrr}
\hline Questão & n & \% \\
\hline Conhece a legislação dos direitos da Pessoa com Deficiência & \\
\hline Sim & 28 & 53,0 \\
Não & 25 & 47,0
\end{tabular}

Prioridade no atendimento nas Estratégias Saúde da Família

\begin{tabular}{lrr}
\hline Pessoa com Deficiência tem prioridade & 41 & 77,4 \\
Pessoa com Deficiência não tem prioridade & 12 & 22,6 \\
\hline
\end{tabular}

Acolhimento da Pessoa com Deficiência nas Estratégias Saúde da Família

\begin{tabular}{lrr}
\hline Não há distinção em relação aos demais usuários & 24 & 45,3 \\
Com prioridade & 20 & 37,7 \\
Respostas descontextualizadas & 9 & 17,0 \\
\hline
\end{tabular}

Diferença na relação/atendimento da Pessoa com Deficiência, pelo tipo de deficiência 
Há diferença

Respostas descontextualizadas

Comunicação com a Pessoa com Deficiência no momento do atendimento

Utilizam estratégia(s), como gestos ou mímicas, escrita, leitura labial e fala pausada

$30 \quad 56,6$

Precisam da ajuda do acompanhante ou conversa diretamente com o familiar, nos casos de $\quad 15 \quad 28,3$ surdos, 'mudos' ou pessoa com deficiência intelectual

Tentam fazê-la de maneira 'normal' ou similar ao diálogo dos demais usuários

59,4

Não têm dificuldades

$3 \quad 5,7$

Situações de necessidade da Pessoa com Deficiência comparecer na Estratégia Saúde da Família com um acompanhante

\begin{tabular}{lrr}
\hline Surdos ou pessoa com deficiência intelectual têm esta necessidade & 36 & 68,0 \\
Menores de 18 anos, idosos ou dependentes de terceiros para uso de medicamentos & 12 & 22,6 \\
Toda Pessoa com Deficiência deve procurar o serviço com algum acompanhante & 5 & 9,4 \\
\hline
\end{tabular}

Fonte: elaborado pelas autoras

A maior parte dos entrevistados alegou nunca ter presenciado alguma atitude/situação de desrespeito às $\operatorname{PcD}$ na $\operatorname{ESF}(77,3 \%)$ e nunca ter ocorrido situações de impossibilidade de atendimento à PcD na ESF (81,1\%) (Tabela 3). Daqueles que relataram impossibilidade, foram citados os motivos: indisponibilidade ou falta de médico ou enfermeiro na equipe, ausência de horários vagos para o atendimento, necessidade de atendimento domiciliar e demandas não referentes à ESF.

Os entrevistados relataram que a relação interpessoal entre a equipe e a $\mathrm{PcD}$ era positiva (86,8\%), assim como também entre a equipe e a família da $\operatorname{PcD}(92,4 \%)$. A maior parte dos trabalhadores negou ter alguma dificuldade ou dúvida sobre o atendimento de $\mathrm{PcD}$ (66\%), afirmou que a mobilidade da PcD até a ESF era ruim ou péssima (92\%), que a unidade não apresenta barreiras na acessibilidade, na área interna ou externa (68\%) (Tabela 3). Os trabalhadores citaram itens que poderiam ser melhorados, como: barras de apoio nos corredores, corrimão nas rampas externas, altura dos bebedouros, piso tátil, entre outros.

Os participantes relataram que a ESF apresentava estrutura física que proporcionava autonomia à $\operatorname{PcD}(81,1 \%)$ e que o atendimento estimulava a independência desta população $(73,6 \%)$ (Tabela 3$)$.

Tabela 3 - Frequência de respostas a respeito das percepções dos trabalhadores das Unidades Básicas de Saúde sobre situações cotidianas do atendimento às Pessoas com Deficiência ( $\mathrm{n}=53)$. Teixeira de Freitas, BA, Brasil, 2017. 


\begin{tabular}{lrr}
\hline Não & 41 & 77,3 \\
Sim & 12 & 22,7 \\
\hline
\end{tabular}

Situações de impossibilidade de atendimento à Pessoa com Deficiência na Estratégia Saúde da Família

\begin{tabular}{|c|c|c|}
\hline Nunca ocorreu & 43 & 81,1 \\
\hline Já ocorreu & 10 & 18,9 \\
\hline \multicolumn{3}{|c|}{ Relação interpessoal entre a equipe e a Pessoa com Deficiência } \\
\hline Positiva & 46 & 86,8 \\
\hline Poderia ser melhor & 6 & 11,3 \\
\hline Não existe relação & 1 & 1,9 \\
\hline \multicolumn{3}{|c|}{ Relação interpessoal entre a equipe e a família da Pessoa com Deficiência } \\
\hline Positiva & 49 & 92,4 \\
\hline Negativa & 4 & 7,6 \\
\hline \multicolumn{3}{|c|}{ Tem alguma dificuldade ou dúvida sobre o atendimento da Pessoa com Deficiência } \\
\hline Não & 35 & 66,0 \\
\hline $\operatorname{Sim}$ & 18 & 44,0 \\
\hline \multicolumn{3}{|c|}{ Mobilidade da Pessoa com Deficiência até a Estratégia Saúde da Família } \\
\hline Ruim ou péssima & 49 & 92,0 \\
\hline Boa ou razoável & 4 & 8,0 \\
\hline \multicolumn{3}{|c|}{ A unidade apresenta barreiras na acessibilidade, na área interna ou externa } \\
\hline Não & 36 & 68,0 \\
\hline $\operatorname{Sim}$ & 17 & 32,0 \\
\hline \multicolumn{3}{|c|}{$\begin{array}{l}\text { A Estratégia Saúde da Família apresenta estrutura física que proporciona autonomia à Pessoa com } \\
\text { Deficiência }\end{array}$} \\
\hline Sim & 43 & 81,1 \\
\hline Não & 10 & 18,9 \\
\hline \multicolumn{3}{|c|}{$O$ atendimento estimula a independência desta população } \\
\hline Sim & 39 & 73,6 \\
\hline Não & 14 & 36,4 \\
\hline
\end{tabular}

Fonte: Elaborado pelas autoras

Além desses questionamentos, foram abordadas outras perguntas para melhor compreensão sobre o atendimento à $\mathrm{PcD}$ nas ESF e a percepção dos trabalhadores sobre este público. Do total de entrevistados, 56,6\% responderam não existir estratégia para incluir as $\mathrm{PcD}$ nos serviços da unidade; e daqueles que afirmaram haver $(43,4 \%)$, as estratégias que estes julgaram existir não são relacionadas especificamente à $\mathrm{PcD}$, consistindo nas ações e programas já ofertados para todos os usuários da ESF.

A maioria dos entrevistados $(85 \%)$ afirmou nunca ter recebido algum treinamento ou capacitação nessa área. Porém 9,4\% relataram que já foi oferecida alguma formação para o 
atendimento de $\mathrm{PcD}$, em momentos anteriores, por parte da gestão municipal. Cerca de 5,6\% não souberam responder a essa questão.

Os trabalhadores relataram não existir recurso adicional para suprir determinada necessidade no atendimento de $\mathrm{PcD}$ (84,9\%). Entretanto, 15,1\% responderam positivamente, citando recursos como: atendimento de assistentes sociais e fisioterapeutas do Núcleo de Apoio à Saúde da Família, oferta de kits de fraldas, curativos, medicamentos, e disponibilidade de cadeira de rodas na unidade.

A maioria dos entrevistados $(75,5 \%)$ relatou não haver qualquer equipamento específico em seu setor para o atendimento de $\mathrm{PcD}$ e $24,5 \%$ afirmaram existir apenas uma cadeira de rodas na sua unidade. De forma similar, 96,2\% dos trabalhadores alegaram não haver nenhuma medida a ser implantada nas unidades para melhorar a acessibilidade e a inclusão das PcD no serviço, mas que medidas deveriam ser estabelecidas, com vistas a melhorar o atendimento a esse público.

Os trabalhadores afirmaram que a $\mathrm{PcD}$ tem retornado à unidade sempre que é necessário um novo atendimento $(86,8 \%)$. No entanto, $13,2 \%$ afirmaram que, às vezes, isso não é possível e quando isso acontece são realizadas visitas domiciliares.

$\mathrm{Na}$ avaliação do próprio atendimento às $\mathrm{PcDs}, 49 \%$ dos entrevistados responderam considerar ser 'bom', 35,9\% 'excelente' e 15,1\% 'nem bom nem ruim'. Em relação à visão sobre as $\mathrm{PcD}, 39,6 \%$ responderam que são 'pessoas com prioridades', ou 'com direito à acessibilidade' ou 'que demandam necessidades especiais e atenção'; 18,9\% responderam 'normal ou igual às demais pessoas'; outras 18,9\% apresentaram respostas relacionadas à exclusão social; 13,2\% responderam 'pessoas que possuem dificuldades'; 7,5\% tiveram respostas descontextualizadas e 1,9\% afirmaram que "mesmo com a 'doença' a PcD pode ser útil”.

A maior parte dos entrevistados $(71,7 \%)$ relatou não haver casos de atendimento de PcD que mora fora da área de cobertura da ESF; entretanto, 28,3\% relataram esse tipo de atendimento. Além disso, 30,2\% relataram que existem PcD moradoras da área de cobertura da ESF mas que não utiliza os serviços da unidade. Segundo estes, os motivos seriam devido a PcD possuir planos de saúde e/ou preferir serviços da rede particular.

Ao final da entrevista, quando indagados sobre alguma questão que ainda não havia sido abordada ou alguma informação a ser acrescentada, os participantes citaram: haver a necessidade de mais ações e estratégias nas unidades, como palestras sobre a temática; mais cuidados centrados na família da $\mathrm{PcD}$, devido ao estresse enfrentado; implantação de um dia 
especifico para o atendimento à $\mathrm{PcD}$; realizar treinamento para $\mathrm{o}$ atendimento às $\mathrm{PcD}$; parcerias entre as redes; além de melhora nas rampas de entrada das unidades.

\section{Discussão}

Quase metade dos participantes alegou não conhecer a legislação sobre os direitos das PcD. Assim, verifica-se que os conhecimentos sobre esta legislação precisam ser mais difundidos, principalmente nos espaços voltados à saúde. Além disso, exemplos mais específicos poderiam ter sido citados pelos profissionais, como: a lei $13146 / 2015$, que institui a lei brasileira de inclusão da pessoa com deficiência (Estatuto da Pessoa com Deficiência) (BRASIL, 2015) e o decreto 5626/2005, que dispõe sobre a Língua Brasileira de SinaisLibras (BRASIL, 2005).

A maior parte dos entrevistados estava ciente sobre a prioridade de $\mathrm{PcD}$ nos serviços, mesmo assim alguns trabalhadores não responderam de forma adequada a essa questão. No entanto, a prioridade a esse público e a avaliação de risco no atendimento em saúde, segundo Art. $9, \S 2^{\underline{0}}$ da lei $13.146 / 2015$, nos serviços de emergência públicos e privados, a prioridade está condicionada aos protocolos de atendimento médico (BRASIL, 2015). As situações distintas encontradas na prática das unidades de saúde, por vezes, podem gerar dúvidas quanto à legitimidade das prioridades e esclarecer essa relativização, de acordo com uma estratificação de riscos faz-se importante nesse contexto, tanto para os profissionais quanto para os usuários do serviço.

A maioria dos entrevistados afirmou que o acolhimento de PcD nas ESF era realizado de maneira igualitária aos demais usuários, ou seja, não propiciava um atendimento equânime. No entanto, o acolhimento à $\mathrm{PcD}$ nos serviços de saúde deve ser especializado e individualizado, considerando as particularidades cada um (GOTADO; ALMEIDA, 2016). O presente resultado corrobora com o evidenciado em outro estudo, onde as $\mathrm{PcD}$ entrevistadas alegaram não se sentirem acolhidas pelos profissionais, não tendo assim sua condição reconhecida e valorizada, o que sugere uma realidade em que alguns profissionais de saúde não têm este entendimento em seu exercício profissional (RESENDE; NÓBREGA; MOREIRA, 2014). Ademais, entendendo o acolhimento como mecanismo de facilitação de acesso, essa ferramenta precisa ser efetivada na Atenção Básica (ALBUQUERQUE et al., 2014), assim como faz-se necessária a implementação do princípio da equidade no SUS (CASTRO et al., 2011). 
De modo semelhante, verificou-se que não havia diferença na relação/atendimento da PcD, pelo tipo de deficiência. No entanto, o cuidado da PcD no SUS, pautado na equidade, trata diferenças e especificidades de maneira singular (CAMPOS; SOUZA; MENDES, 2015). Assim, para o serviço de saúde equânime, torna-se fundamental reconhecer as diferenças no coletivo e enfrentar os preconceitos e estigmas que ainda atingem esta população.

Os profissionais relataram o uso de estratégia(s), como gestos ou mímicas, escrita, leitura labial e fala pausada para comunicação com a PcD no momento do atendimento. Contudo, estratégias de comunicação (escrita, leitura labial e a presença do acompanhante) com usuários surdos usadas pelos profissionais de saúde podem ser ineficientes, além não estimulam a participação ativa dos pacientes. Além disso, para pacientes surdos, o atendimento realizado por profissionais que saibam Libras ou em locais que oferecem intérprete mantém a sua privacidade e independência (OLIVEIRA; CELINO; COSTA, 2015). Em consonância, uma pesquisa sobre dificuldades no atendimento da pessoa surda por profissionais da saúde de ESF ressaltou que a dificuldade de comunicação constitui uma barreira para a promoção de saúde (GIL DE FRANÇA et al., 2016).

Embora um menor percentual de entrevistados tenha relatado já ter presenciado situações de desrespeito às $\mathrm{PcD}$, tais situações precisam ser erradicadas da sociedade. Visto que as barreiras que impedem a inclusão das $\mathrm{PcD}$ nos diversos serviços são não apenas arquitetônicas, mas sim comportamentais, tais problemáticas provocam a violação dos direitos destas pessoas (PEREIRA; MEDEIROS, 2016).

A maioria dos profissionais relatou uma boa relação com a $\mathrm{PcD}$ e com seus familiares. No entanto, resultados contrários foram verificados em outro estudo, que questionou familiares, pois a maioria dos entrevistados relataram que, raramente, se sentiam respeitados nos serviços das unidades de saúde (GOTADO; ALMEIDA, 2016). Sendo assim, é relevante valorizar a satisfação da PcD e/ou da sua família, por meio da escuta dos usuários, com vistas ao fortalecimento de vínculos com a equipe e à resolutividade dos serviços em saúde.

A mobilidade da PcD até a ESF foi indicada como ruim ou péssima pelos profissionais. Em consonância ao encontrado, também foi verificado condições insatisfatórias de acessibilidade nas vias públicas e nenhuma acessibilidade às edificações para idosos nas unidades de Atenção Primária à Saúde de outro estado do Nordeste brasileiro (PAGLIUCA et al., 2017). Assim, destaca-se a importância de uma infraestrutura adequada das vias públicas, para possibilidades de acesso das PcD, ou pessoas com mobilidade reduzida, aos serviços de saúde. 
Os participantes afirmaram que as unidades não apresentavam barreiras na acessibilidade, com estruturas físicas que proporcionavam autonomia à $\mathrm{PcD}$. Porém, os altos percentuais encontrados sobre acessibilidade física já eram esperados, uma vez que as unidades estudadas foram planejadas e construídas para um acesso universal. À exceção das barras de apoio nos corredores, os demais itens citados pelos entrevistados que poderiam ser melhorados nas ESF - corrimão nas rampas externas, altura dos bebedouros, piso tátil, entre outros, estão previstos na ABNT-NBR 9050/2015. Isso revela uma boa visão dos profissionais sobre as normatizações do ambiente para propiciar a acessibilidade às $\mathrm{PcD}$, assim como sugere uma possibilidade de analisar a normatização das barras de apoio nos corredores, tanto nos espaços de atendimento das ESF, quanto nos demais espaços urbanos. Não obstante, outros estudos verificaram que a acessibilidade nos serviços de saúde no Brasil ainda está prejudicada (ALBUQUERQUE et al., 2014; CAASTRO et al., 2011; CRUZ et al., 2019; RIBEIRO et al., 2015; SILVA et al., 2015). Porém, com a implantação do Programa Nacional de Melhoria do Acesso e da Qualidade da Atenção Básica, profissionais da área da saúde perceberam melhorias de acesso para PcD às ESF (MOCELIN et al., 2017).

Embora a maioria dos profissionais relatou uma visão de $\mathrm{PcD}$ como pessoas com prioridades, direito à acessibilidade ou com necessidades especiais, uma parte considerável apresentou respostas de igualdade às demais pessoas ou relacionadas à exclusão social. Muitos estigmas relacionados a essa população ainda perpetuam nos dias atuais (MAGALHÃES; CARDOSO, 2010), o que gera preconceito e negação social, pois tratam a deficiência como uma "falta, carência ou impossibilidade", concepções presentes na sociedade ao longo da história da humanidade (COSTA, 2009).

Relativo aos equívocos observados acerca da identificação dos tipos de deficiência, o que gerou enganos quanto aos pacientes com problemas psiquiátricos, acamados, domiciliados, com Alzheimer e usuários acometidos por Acidente Vascular Encefálico. Essa falta de esclarecimento sobre as particularidades que diferenciam um paciente com e sem deficiência ocasiona prejuízos na assistência oferecida pelos profissionais de saúde (COSTA, 2011).

Quase metade dos profissionais afirmaram ter alguma dificuldade ou dúvida sobre o atendimento de $\mathrm{PcD}$ e apenas um terço, avaliou o próprio atendimento às $\mathrm{PcD}$ como excelente. Esses achados indicam a necessidade de uma capacitação profissional continuada, e específica sobre o tema em questão, além de corroborar com as sugestões dos participantes ao final da entrevista. Recursos financeiros e profissionais precisam ser destinados a melhoria dos atendimentos às $\mathrm{PcD}$, como, por exemplo para capacitações aos profissionais, intérprete 
de libras, dispositivos em braile nos serviços, piso tátil, entre outros. Destaca-se a importância da capacitação e da qualificação dos trabalhadores para o atendimento a esse público (BEZERRA; SILVA; MAIA, 2015; BOWONIUK WIEGAND; LEAL DE MEIRELLES, 2019; MAIA et al., 2009).

\section{Considerações finais}

A percepção e o conhecimento dos profissionais das UBSs sobre acessibilidade e assistência da PcD nos serviços de saúde ainda precisam ser aprimorados, tendo em vista que metade dos profissionais não conheciam a legislação. Alguns profissionais afirmaram que o acolhimento era realizado de maneira igualitária aos demais usuários - não propiciando a equidade, assim com outros relataram dúvidas relacionadas ao atendimento a essa população.

Para isso, deve-se oferecer condições favoráveis à equipe, tendo em vista as dificuldades a serem enfrentadas para se obter um cuidado resolutivo, de forma a valorizar a autonomia e a independência das $\mathrm{PcD}$, o acesso facilitado, a comunicação eficiente com profissionais e o atendimento de qualidade.

AGRADECIMENTOS: Ao Programa Institucional de Bolsas de Iniciação à Pesquisa, Criação e Inovação, da Universidade Federal do Sul da Bahia (PIBIC-UFSB).

\section{REFERÊNCIAS}

ALBUQUERQUE, M. S. V. et al. Acessibilidade aos serviços de saúde: uma análise a partir da Atenção Básica em Pernambuco. Saúde em debate, v. 38, n. esp., p. 182-194, out. 2014.

ASSOCIAÇÃO BRASILEIRA DE NORMAS TÉCNICAS. ABNT NBR 9050:

acessibilidade a edificações, mobiliário, espaços e equipamentos urbanos. 3. ed. Rio de Janeiro: Associação Brasileira de Normas Técnicas, 2015.

BEZERRA, T. V.; SILVA, M. A.; MAIA, E. R. Acesso da pessoa com deficiência à atenção primária no Brasil: limites e possibilidades. Cadernos de Cultura e Ciência, v. 2, n. 14, p. 65-74, dez. 2015.

BOWONIUK WIEGAND, B.; LEAL DE MEIRELLES, J. M. Saúde das pessoas com deficiência no Brasil: uma revisão integrativa na perspectiva bioética. Revista

Latinoamericana de Bioética, v. 19, n. 2, p. 29-44, dez. 2019.

BRASIL. Casa Civil. Lei n. 13.146, de 6 de julho de 2015. Institui a Lei Brasileira de Inclusão da Pessoa com Deficiência (Estatuto da Pessoa com Deficiência). Diário Oficial da União: Seção 1, Brasília, DF, p. 2, 07 jul. 2015. PL 7699/2006 
BRASIL. Decreto n. 5.626, de 22 de dezembro de 2005. Regulamenta a Lei n. 10.436, de 24 de abril de 2002, que dispõe sobre a Língua Brasileira de Sinais - Libras, e o art. 18 da Lei n. 10.098, de 19 de dezembro de 2000. Brasília, DF, 23 dez. 2005. Disponível em:

BRASIL. Decreto n. 7.612, de 17 de novembro de 2011. Institui o Plano Nacional dos Direitos da Pessoa com Deficiência - Plano Viver sem Limite. Brasília, DF, 18 nov. 2011. Disponível em: http://www.planalto.gov.br/ccivil_03/_ato20112014/2011/decreto/d7612.htm. Acesso em: 09 ago. 2020.

BRASIL. Ministério da Saúde. Portaria n. 793, de 24 de abril de 2012. Institui a Rede de Cuidados à Pessoa com Deficiência no âmbito do Sistema Único de Saúde. Brasília, DF: Ministério da Saúde, 25 abr. 2012. Disponível em:

http://bvsms.saude.gov.br/bvs/saudelegis/gm/2012/prt0793_24_04_2012.html. Acesso em: 09 ago. 2020.

BRASIL. Ministério da Saúde. Portaria n ${ }^{0}$ 2.436, de 21 de setembro de 2017. Aprova a Política Nacional de Atenção Básica. Diário Oficial da União: Seção 1, Brasília, DF, p. 68, 22 set. 2017. Disponível em: https://www.in.gov.br/materia/-

/asset_publisher/Kujrw0TZC2Mb/content/id/19308123/do1-2017-09-22-portaria-n-2-436-de21-de-setembro-de-2017-19308031. Acesso em: 09 ago. 2020.

CAMPOS, M. F.; SOUZA, L. A. P.; MENDES, V. L. F. A rede de cuidados do Sistema Único de Saúde à saúde das pessoas com deficiência. Interface, Botucatu, v. 19, n. 52, p. 207-210, jan./mar. 2015.

CASTRO, S. S. et al. Acessibilidade aos serviços de saúde por pessoas com deficiência. Revista de Saúde Pública, v. 45, n. 1, p. 99-105, fev. 2011.

COSTA, K. N. F. M. Modelo de comunicação verbal com o cego: desenvolvimento e validação em consulta de enfermagem. 2009. 131 f. Tese (Doutorado em Enfermagem) Faculdade de Farmácia, Odontologia e Enfermagem, Universidade Federal do Ceará, Fortaleza, 2009.

COSTA, L. S. M. Educação médica e atenção integral à saúde da pessoa com deficiência. Revista Brasileira de Educação Médica, v. 35, n. 3, p. 301-302, jul./set. 2011.

CRUZ, R. et al. Acessibilidade para pessoas com deficiência na atenção básica de saúde em Três Rios, Centro Sul Fluminense. Enfermagem Brasil, v. 18, n. 1, p. 95-104, 2019.

GIL DE FRANÇA, E. et al. Dificuldades de profissionais na atenção à saúde da pessoa com surdez severa. Ciencia y enfermeira, v. 22, n. 3, p. 107-116, set. 2016. Disponível em: https://scielo.conicyt.cl/pdf/cienf/v22n3/0717-9553-cienf-22-03-00107.pdf. Acesso em: 09 ago. 2020 .

GOTADO, R. G.; ALMEIDA, M. A. P. T. O Acolhimento oferecido pelos Serviços de Saúde às pessoas com Necessidades Especiais. Id on Line Revista Multidisciplinar e de Psicologia, v. 10, n. 32, p. 126-137, nov./dez. 2016. Disponível em: https://idonline.emnuvens.com.br/id/article/view/568/789. Acesso em: 09 ago. 2020. 
http://www.planalto.gov.br/ccivil_03/_ato2004-2006/2005/decreto/d5626.htm. Acesso em: 09 ago. 2020.

MAGALHAES, R. C. B. P.; CARDOSO, A. P. L. B. A pessoa com deficiência e a crise das identidades na contemporaneidade. Cadernos de Pesquisa, v. 40, n. 139, p. 45-61, jan./abr. 2010.

MAIA, E. R. et al. Assistência à pessoa com deficiência: competências do agente comunitário de saúde. Revista de Enfermagem UFPE on-line, v. 3, n. 4, p. 937-944, out./dez. 2009. Disponível em: https://pdfs.semanticscholar.org/9bcc/3bb3555385bad6afeaf43ba92fc92b2dfaaf.pdf. Acesso em: 09 ago. 2020.

MOCELIN, G. et al. Melhoria do acesso e da qualidade na atenção básica em saúde: inserção da pessoa com deficiência. Cinergis, v. 18, p. 353-357, dez. 2017. DOI:

https://online.unisc.br/seer/index.php/cinergis/article/view/11165/7149

OLIVEIRA, Y. C. A; CELINO, S. D. M.; COSTA, G. M. C. Comunicação como ferramenta essencial para assistência à saúde dos surdos. Physis: Revista de Saúde Coletiva, v. 25, n. 1, p. 307-320, jan./mar. 2015.

PAGLIUCA, L. M. F. et al. Acesso de idosos às unidades de Atenção Primária à Saúde. REME - Revista Mineira de Enfermagem, v. 21, n. e1021, p. 1-5, set. 2017.

PEDRAZA, D. F. et al. Acessibilidade às unidades básicas de saúde da família na perspectiva de idosos. Ciência \& Saúde Coletiva, v. 23, p. 923-933, 2018.

PEREIRA, N. T. B.; MEDEIROS, R. A. A Realidade das Agências Bancárias de João PessoaPB: Entraves à acessibilidade para Pessoas com Deficiência. Revista de Direito Sociais e Políticas Públicas, v. 2, n. 2, p. 37-51, jul./dez. 2016.

RESENDE, A. C.; NÓBREGA, S.; MOREIRA, T. Experiência das Pessoas com Deficiência nos Serviços de Saúde: Contribuição para a Formação Profissional. In: CONGRESSO INTERNACIONAL DE HUMANIDADES \& HUMANIZAÇÃO EM SAÚDE, São Paulo, 2014. Anais [...]. São Paulo, SP: Blucher, 2014. p. 61.

RIBEIRO, J. P. et al. Acessibilidade aos serviços de saúde na Atenção Básica do Estado de Goiás. Revista Eletrônica de Enfermagem, v. 17, n. 3, p. 1-11, jul./set. 2015. Disponível em: https://www.fen.ufg.br/fen_revista/v17/n3/pdf/v17n3a04.pdf. Acesso em: 09 ago. 2020.

SILVA, D. C. N. et al. Acessibilidade de portadores de deficiência física ou mobilidade reduzida na Unidade Básica de Saúde Jonas Manoel Dias em São Luís de Montes Belos GO. Revista Faculdade Montes Belos (FMB), v. 8, n. 3, p. 36-60, nov. 2015. 


\section{Como referenciar este artigo}

SEDLMAIER, B. M. G.; MOURÃO, D. M.; FERREIRA, C. G.; SILVEIRA, C. L. G.; BORGES, G. F. Percepção e conhecimento de profissionais de unidades básicas da saúde sobre acessibilidade das pessoas com deficiência. Temas em Educ. e Saúde, Araraquara, v. 17, n. $00, \quad$ p. e021003, 2021. e-ISSN 2526-3471. DOI: https://doi.org/10.26673/tes.v17i00.14251

Submetido em: 30/09/2020

Revisões requeridas: $28 / 11 / 2020$

Aceito em: 23/03/2021

Publicado em: 20/04/2021 